\title{
Estimating the thickness of diffusive solid electrolyte interface
}

\author{
XiaoHe Wang ${ }^{1,2}$, WenHao Shen ${ }^{1,2}$, XianFu Huang ${ }^{1,2}$, JinLiang Zang ${ }^{1}$, and YaPu Zhao ${ }^{1,2^{*}}$ \\ ${ }^{1}$ State Key Laboratory of Nonlinear Mechanics, Institute of Mechanics, Chinese Academy of Sciences, Beijing 100190, China, \\ ${ }^{2}$ School of Engineering Science, University of Chinese Academy of Sciences, Beijing 100049, China
}

Received March 21, 2017; accepted April 1, 2017; published online April 19, 2017

\begin{abstract}
The solid electrolyte interface (SEI) is a hierarchical structure formed in the transition zone between the electrode and the electrolyte. The properties of lithium-ion (Li-ion) battery, such as cycle life, irreversible capacity loss, self-discharge rate, electrode corrosion and safety are usually ascribed to the quality of the SEI, which are highly dependent on the thickness. Thus, understanding the formation mechanism and the SEI thickness is of prime interest. First, we apply dimensional analysis to obtain an explicit relation between the thickness and the number density in this study. Then the SEI thickness in the initial charge-discharge cycle is analyzed and estimated for the first time using the Cahn-Hilliard phase-field model. In addition, the SEI thickness by molecular dynamics simulation validates the theoretical results. It has been shown that the established model and the simulation in this paper estimate the SEI thickness concisely within order-of-magnitude of nanometers. Our results may help in evaluating the performance of SEI and assist the future design of Li-ion battery.
\end{abstract}

lithium-ion battery, solid electrolyte interface, diffusion model, thickness estimation

PACS number(s): 66.30.-h, 82.47.Aa, 68.35.Md, 02.70.Ns

Citation: X. H. Wang, W. H. Shen, X. F. Huang, J. L. Zang, and Y. P. Zhao, Estimating the thickness of diffusive solid electrolyte interface, Sci. China-Phys. Mech. Astron. 60, 064612 (2017), doi: 10.1007/s11433-017-9031-2

\section{Introduction}

The core technology of secondary lithium-ion (Li-ion) batteries is the use of intercalation materials as electrodes. The $\mathrm{LiMnO}_{2}$ as the current commercial cathode active material has a high Li-ion intercalating and de-intercalating potential, and the graphite material with a low Li-ion intercalating and de-intercalating potential is the negative material [1]. Electrochemical reactions forming a solid electrolyte interface (SEI) film will take place on the electrode surface during the initial charge-discharge cycle [2]. The SEI formation is accompanied by the decomposition of the organic electrolyte and the intercalation of lithium (Li) ions in the electrode. The SEI is a complete, dense and passive film that separates the

"Corresponding author (email: yzhao@imech.ac.cn) electrolyte from the electrode. In general, a SEI has two functions. First, forming a SEI consumes Li ions of the battery in the electrochemical reactions, which leads to the decline of the battery effective capacity. However, the SEI generated by the reactions will prevent further erosion of the electrode during subsequent electrochemical cycles and maintain the stability of battery anodes $[3,4]$. Second, the SEI has the ionic conductivity, while it acts as an electrical insulator. Thus, the SEI affects Li-ion battery electrochemical cycle performance, cycle life, safety, etc. [5,6]. The most commonly used experimental methods to observe the SEI are summarized and discussed in Table 1 [7-14].

Because of the complexity of both electrolyte compositions and surface morphology of the electrode, studies on the compositions, property and thickness of the SEI have been hotspots in the research of secondary Li-ion batteries [15-20]. The electrochemical models in cells for the graphite anodes 
Table 1 The summary of the observing method of the SEI

\begin{tabular}{|c|c|}
\hline Method & Observation \\
\hline Electrochemical Impedance Spectroscopy (EIS) [7] & The SEI forming and decomposition process on $\mathrm{Li}$ anodes \\
\hline Cyclic Voltammetry Measurements (CV) [8] & The potential range of the SEI film formation on graphite anodes \\
\hline X-ray Photoelectron Spectroscopy (XPS) [9] & The presence of the SEI and its element compositions \\
\hline Scanning and Transmission Electron Microscopy (SEM-TEM) [9] & A plastic and porous layer on the surface of $\mathrm{Li}$ and aluminum electrodes \\
\hline In Situ X-Ray Diffraction (XRD) [10] & $\mathrm{Li}-\mathrm{C}$ intercalation processes \\
\hline In Situ Raman Spectroscopy [12] & $\begin{array}{c}\text { Changes in the surface crystallinity of graphite negative- } \\
\text { electrodes by different electrolytes }\end{array}$ \\
\hline Fourier Transform Infrared Spectrometer (FT-IR) [13] & Ions of the compositions in the SEI, such as $\mathrm{CO}_{3}{ }^{2-}, \mathrm{ClO}_{4}^{-}$, and $\mathrm{Cl}^{-}$ \\
\hline Pyrolysis/Gas Chromatography/Mass Spectroscopy (GC-MS) [14] & The accurate compositions of the SEI \\
\hline
\end{tabular}

with different electrolyte compositions (PC, EC, DMC, DEC $+\mathrm{LiPF}_{6}$ ) were discussed [21-23]. Generally, the SEI is mainly composed of $\mathrm{Li}_{2} \mathrm{CO}_{3}$, $\mathrm{LiF}$, other simple Li salts, $\mathrm{Li}_{2} \mathrm{O}$, Li alkyl carbonates, Li alkyl oxides and polymer components on the surface of the graphite-based electrode. On one hand, the SEI needs to have a good Li-ion conductivity as an excellent $\mathrm{Li}$-ion channel to accelerate the intercalation reaction at the electrode. On the other hand, the SEI needs to be stable enough to prevent the irreversible loss of Li-ion. As a result, it plays a fundamental role in increasing the Li storage capacity of the battery to form a SEI with stable electrochemical properties and a uniform thickness.

The silicon ( $\mathrm{Si}$ ) electrode, as a research focus of the $\mathrm{Li}$-ion batteries, has a higher capacity than the graphite anode. However, few investigations focused on the forming mechanism and electrochemical properties of the SEI on the Si electrode surface whose research is still at the qualitative stage [24,25]. It was deemed no SEI formed on the Si surface in early studies. On one hand, a large amount of irreversible capacity loss was obtained by observing the reversible capacity during charge-discharge cycles, and the detachment of the electrode from the current collector during the discharge was observed [26]. It was considered that the irreversible capacity loss at the Si electrode was due to the incomplete discharge caused by the detachment of the intercalation electrode [4,26,27]. On the other hand, the SEI structure on the Si surface was similar to that on the graphite surface, which was not observed before. The disappearing of the SEI structure may be caused by the drastic change of electrode surface morphology [26,28]. The electrode cracked and a new electrolyte interface was formed during the intercalation of the Si electrode simultaneously. Li salts on the Si surface were similar to those on the graphite surface. This phenomenon was observed using X-ray photoelectron spectroscopy (XPS) [24]. The morphology of the SEI on the Si substrate was also observed with scanning electron microscopy (SEM) [29,30].

Due to the deformation of the Si electrode during the intercalation, the point located in the performance and the thickness SEI on the Si surface becomes a difficulty [31]. In this paper, a theoretical model for the thickness estimation of the SEI is presented using the Cahn-Hilliard phase-field model. To verify the results of the magnitude estimation, molecular dynamics (MD) simulation is carried out.

\section{Theoretical model of the SEI thickness}

The SEI formation on the electrode surface mainly completes in the initial charge-discharge cycle of Li-ion battery. The reaction and diffusion are the dominant mechanisms at this stage. Thus, the concentration of Li-ion, the bond strength, the number density of Li-ion at the interface, the temperature and the energy barrier are dominant physical quantities that influence the SEI thickness. The dimensional analysis (DA) is done in the Appendix giving a preliminary result about the SEI thickness, as shown in eq. (a2). The DA result plays a guidance function for the theoretical analysis. In addition, there is an implicit relation between the dominant physical quantities and the SEI thickness, e.g. the thickness is in inverse proportion to the square root of number density of Li-ion that is the number of $\mathrm{Li}$-ion per unit area. However, using DA, there is only an indefinite function $f$, which implies that the solution is incomplete. A theoretical model is established as follows to obtain further results.

Considering the similarity between the SEI formation and the typical diffusion interface structure, the Cahn-Hilliard phase-field model is used to estimate the SEI thickness. The Li-ion is selected as the solvent-ion and the Si electrode as the solid solution. In this study, the SEI forming mechanism is investigated using the generalized diffusion theory that the chemical potential drives the diffusion of ions.

According to the Fick's first law, the Li-ion flux density $j$ of the one-dimensional (1D) diffusion system is [32,33]

$$
\begin{aligned}
j & =-M \frac{\partial}{\partial x}\left(\mu_{\mathrm{B}}-\mu_{\mathrm{A}}\right) \\
& =-M \frac{\partial}{\partial x} \frac{\partial g(c)}{\partial c}=-M \frac{\partial^{2} g(c)}{\partial c^{2}} \frac{\partial c}{\partial x}
\end{aligned}
$$


where $M$ is the Li-ion mobility, $\mu_{\mathrm{B}}$ and $\mu_{\mathrm{A}}$ are the Li-ion chemical potentials in electrolyte and the $\mathrm{Si}$ anode, respectively, $g$ is Helmholtz free energy density of Li-ion and $c$ is the Li-ion concentration. According to the Fick's second law, the concentration $c$ is related to the flux density $j$ as:

$\frac{\partial c}{\partial t}+\frac{\partial j}{\partial x}=0$

and the general diffusion equation with the Helmholtz free energy of Li-ion, by substituting eq. (1) into eq. (2), is given as:

$$
\begin{aligned}
\frac{\partial c}{\partial t} & =\frac{\partial}{\partial x}\left(M \frac{\partial^{2} g(c)}{\partial c^{2}} \frac{\partial c}{\partial x}\right) \\
& =M\left[\frac{\partial^{2} g(c)}{\partial c^{2}} \frac{\partial^{2} c}{\partial x^{2}}+\frac{\partial^{3} g(c)}{\partial c^{3}}\left(\frac{\partial c}{\partial x}\right)^{2}\right] \\
& =M \frac{\partial^{2} g(c)}{\partial c^{2}} \frac{\partial^{2} c}{\partial x^{2}}
\end{aligned}
$$

which is the Cahn-Hilliard equation and neglects the second term in the brackets as $(\partial c / \partial t)^{2}$ is the second-order small quantity. With the expansion of free energy by taking the concentration into consideration, eq. (3) becomes a typical fourth-order nonlinear diffusion equation when the chemical potential drives the material transport. It is also the control equation of phase-field dynamics. Eq. (3) can be applied to the fragmentation study, phase transition simulation and interface thickness estimation when stress and chemical fields are coupled synthetically [33]. When eq. (3) is applied to the SEI evolutionary study, the free energy of Li-ion at the SEI is influenced by relevant physical quantities. The choice of the quantities depends on the stages of charge-discharge cycles. One can use the total Helmholtz free energy $F_{\text {tot }}$ to consider the combined effect of $\mathrm{Li}$-ion diffusion and other phenomena. For example, according to the SEI evolutionary behavior on the Si electrode during the intercalation, we need to consider the combined effect of the bulk free energy $F_{\text {bulk }}$, the interface free energy $F_{\text {inter, }}$, the elastic strain energy $F_{\text {elast }}$, etc. The interface free energy is generated in the SEI when the Li salts with different components formed. The substrate deformation introduces the elastic strain energy of the Si electrode. As the Li-ion diffusion coupled with multifold effects, we can get the total Helmholtz free energy as:

$F_{\text {tot }}=F_{\text {bulk }}+F_{\text {inter }}+F_{\text {elast }}+\cdots$.

Figure 1 shows the reaction model of the Si electrode, the SEI and the electrolyte. Due to the deficiency of Li-ion in the $\mathrm{Si}$ electrode in the initial cycle, Li ions diffuse into the $\mathrm{Si}$ electrode from the electrolyte or Li electrode during the SEI formation. The compositions of the SEI on the graphite electrode, which have different salts, exist near the electrolyte and the Si electrode. In this study, we take Li-ion as the labeled ion of the interface formation, and denote the concentration
Anode (Si) Electrolyte $-\mathrm{Li}^{+}$ (a)

(b)

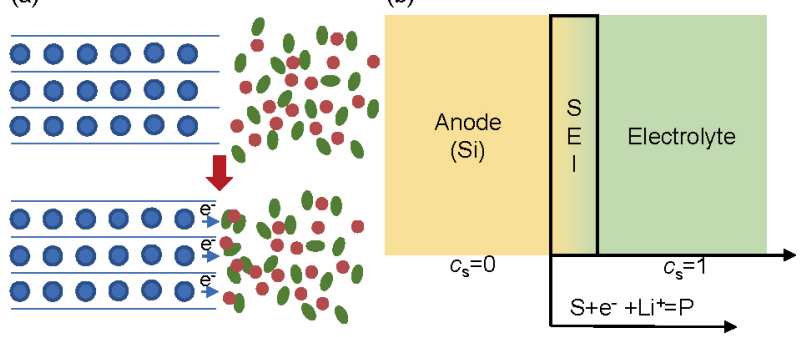

Figure 1 (Color online) (a) The reaction schematic at the interface; (b) the diffusion interface schematic on the electrode surface.

of Li-ion as $c$. The concentration in the Si electrode is zero before the interface formed and one in the electrolyte solution and the Li electrode.

The evolutionary dynamics on the elastic substrate is similar to that on the two-phase monolayer [34]. Since the SEI formation is influenced by the substrate, the free energy $F_{\text {tot }}$ of Li-ion diffusion on the Si electrode surface is determined by the bulk deformation and the interface free energy. The free energy $F_{\text {tot }}$ is defined as:

$F_{\mathrm{tot}}=\int_{A} \Gamma \mathrm{d} A+\int_{V} W \mathrm{~d} V$

where $\Gamma$ is the free energy density of Li-ion at the interface, and $W$ is the strain energy density of the bulk portion. The corresponding integrals of $\Gamma$ and $W$ are completed at the electrode surface $A$ and the electrode body phase $V$, respectively. The SEI formation is mainly completed in the initial charge-discharge cycle. There are few $\mathrm{Li}$ ions embedding in the electrode in the initial charge-discharge cycle, so the deformation of the Si electrode at the body part can be neglected. During the subsequent intercalation process, the SEI would deform and crack with the dramatic change of Si electrode volume. Thus, it is necessary to consider the volumetric strain energy density of electrode for the evolution of the SEI in the subsequent cycles, rather than the first cycle. Therefore, the second term on the right-hand side of eq. (5) is negligible at the SEI formation stage.

The evolution of the free energy density of the SEI is expressed, with the first-order expansion of $\Gamma$ in the concentration [34], as:

$\Gamma=g+h \frac{\partial c}{\partial x_{\beta}} \frac{\partial c}{\partial x_{\beta}}+k \varepsilon_{\beta \beta} \quad(\beta=1,2,3)$,

where $g$ is the free energy density of Li-ion in the interface between the Si electrode and electrolyte, $\partial c / \partial x_{\beta}$ is the gradient term of Li-ion concentration, $\varepsilon_{\beta \beta}$ is the volume strain of the Si electrode, and $h$ and $k$ are the corresponding expansion coefficients. According to Einstein summation convention, summation is taken over repeated index $\beta$. Note that the interface is considered as a regular solution. The strain is caused mainly by the intercalation. Defining $J_{\beta}$ and $\Lambda$ as the number 
flux and the number density, respectively, eq. (2) leads to

$\frac{\partial c}{\partial t}=-\frac{\partial J_{\beta}}{\Lambda \partial x_{\beta}}$,

while Suo and $\mathrm{Lu}$ [35] also define a physical quantity $I_{\beta}$, the expression between $I_{\beta}$ and $J_{\beta}$ is $\partial I_{\beta} / \partial t=J_{\beta}$, so that eq. (7) can be rewritten as:

$\delta c=-\frac{1}{\Lambda} \delta \frac{\partial I_{\beta}}{\partial x_{\beta}}$.

Because of the absence of the body-phase deformation in the electrode during the initial cycle, the effect of the bulk volume strain of the Si electrode is negligible in the expression of the SEI free energy $F_{\text {tot. }}$. Based on the variational principle, the evolution of SEI free energy expression at the initial interface is

$$
\begin{aligned}
\delta F_{\text {tot }} & =\int \frac{\partial}{\Lambda \partial x_{\beta}}\left(\frac{\partial g}{\partial c}-2 h_{0} \nabla^{2} c\right) \delta I_{\beta} \mathrm{d} A \\
& =-\int P_{\beta} \delta I_{\beta} \mathrm{d} A,
\end{aligned}
$$

where $h_{0}$ is assumed as the energy barrier, $h=h_{0}$, and $P_{\beta}$ is the gradient of chemical potential that acts as the driving force. There is a linear function between $J_{\beta}$ and $P_{\beta}$ as [36]:

$J_{\beta}=M P_{\beta}$.

The diffusion equation of Li-ion in the initial charge-discharge cycle is obtained by combining eqs. (8)-(10) as:

$\frac{\partial c}{\partial t}=\frac{M}{\Lambda^{2}} \nabla^{2}\left(\frac{\partial g}{\partial c}-2 h_{0} \nabla^{2} c\right)$,

where the first and second terms in the parentheses indicate the gradient of driving energy density required by the Li-ion diffusion and the resistance in this process, respectively. When the interface approaches the final equilibrium $(\partial c / \partial t=0)$, the steady-state distribution equation of Li-ion concentration is

$\nabla^{2}\left(\frac{\partial g}{\partial c}-2 h_{0} \nabla^{2} c\right)=0$.

On the basis of the free energy, eq. (12) means that the driving energy of Li-ion diffusion competes with the energy barrier encountered by diffusion. When the equilibrium is reached, reactions forming SEI cannot occur continually, so a stable SEI forms.

\section{Molecular dynamics simulation}

MD simulation was carried out to validate the theoretical model, and to help in further understanding the forming mechanism of the SEI. We established a model with two interfaces, as shown in Figure 2, to eliminate the effect of the boundary conditions in the simulation. Both sides of the system were $\mathrm{Si}$, and the middle was $\mathrm{Li}$. The system included $126364 \mathrm{Li}$ atoms and $144000 \mathrm{Si}$ atoms. The simulation was performed using Large-scale Atomic/Molecular Massively Parallel Simulator (LAMMPS). The time step length for the simulation was $1 \mathrm{fs}$. To ensure a sufficient balance of the system, we set the total relaxation to 106 steps. After the system achieved equilibrium sufficiently, every 100 simulation steps were counted once, and we got the final average of the thickness after counting 100 values.

Constant number of atoms, pressure and temperature (NPT) ensemble and the Nose-Hoover thermocouple algorithm (NHTA) were performed for the simulation at $300 \mathrm{~K}$. Each Si atom takes one unit negative charge and each $\mathrm{Li}$ atom has one unit positive charge [37]. The Li-Si interatomic interaction is based on the second nearest-neighbor modified embedded atom method (2NN MEAM) [38-40]. The energy of single atom is

$E^{u}(R)=F\left[\bar{\rho}^{0}(R)\right]+\frac{Z_{1}}{2} \varphi(R)+\frac{Z_{2} S}{2} \varphi(a R)$,

where $F$ is the embedding function, $Z_{1}$ and $Z_{2}$ are the numbers of nearest-neighbor atoms and second nearest-neighbor atoms, $\bar{\rho}^{0}$ is the background electron density of the reference structure, $S$ and $a$ are constants in each structure for a given value of $C_{\min }$. The force-field parameters of Li-Si alloy listed in the Tables 2 and 3 are adopted for the simulation $[41,42]$.

\section{Result and discussion}

The binary system is applied to estimate the thickness and simplify the model as follows. The SEI is assumed as a regular solution similar to the real condition. Based on the regular solution theory, the expression of free energy density $g$ in the binary system is expressed as [22]:

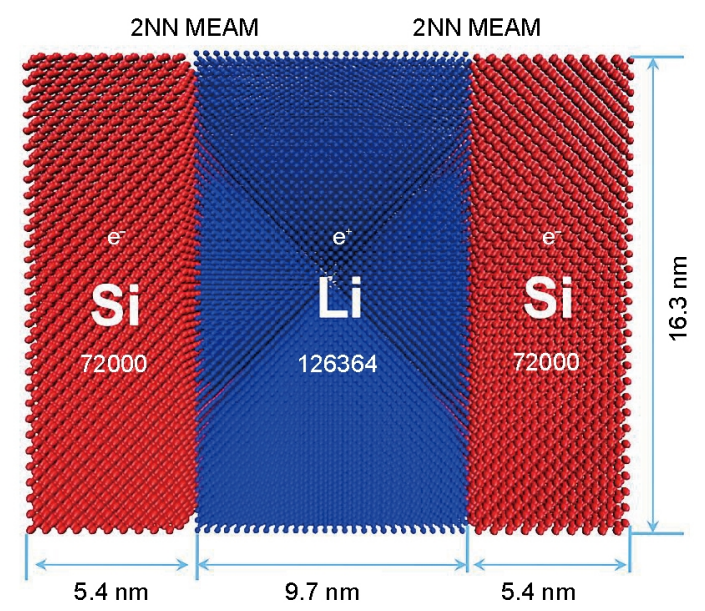

Figure 2 (Color online) Schematic of the diffusion interface model in MD simulation. 
Table 2 Parameters of the Si and Li elements in the simulation

\begin{tabular}{ccccccccccccccccc}
\hline & $E_{\mathrm{c}}(\mathrm{eV})$ & $r_{\mathrm{e}}(\AA)$ & $\alpha$ & $d$ & $A$ & $\beta^{(0)}$ & $\beta^{(1)}$ & $\beta^{(2)}$ & $\beta^{(3)}$ & $t^{(1)}$ & $t^{(2)}$ & $t^{(3)}$ & $C_{\max }$ & $C_{\min }$ \\
\hline $\mathrm{Si}$ & 4.63 & 2.35 & 4.90 & 0.03 & 0.53 & 3.00 & 7.50 & 0.00 & 3.00 & 1.45 & 7.61 & -2.10 & 2.60 & 0.75 & \\
$\mathrm{Li}$ & 1.65 & 2.99 & 3.00 & 0.14 & 0.64 & 1.03 & 4.88 & 4.15 & 5.27 & -1.46 & 4.13 & -0.57 & 1.91 & 0.31 \\
\hline
\end{tabular}

Table 3 Parameters of the Li-Si alloy in the simulation

\begin{tabular}{ccccc}
\hline$E_{\mathrm{c}}(\mathrm{eV})$ & $r_{\mathrm{e}}(\AA)$ & $\alpha$ & $D$ & $\rho_{0}\left(\rho_{0}{ }^{\mathrm{Si}} / \rho_{0}{ }^{\mathrm{Li}}\right)$ \\
\hline 2.45 & 2.75 & 4.10 & 0.10 & 3.0 \\
& Li-Li-Si & Si-Si-Li & Li-Si-Li & Li-Si-Si \\
$C_{\max }$ & 2.81 & 2.20 & 2.40 & 2.40 \\
$C_{\min }$ & 0.55 & 0.35 & 0.45 & 0.45 \\
\hline
\end{tabular}

$$
\begin{aligned}
g= & g_{\mathrm{Li}} c+g_{\mathrm{Si}}(1-c) \\
& +\Lambda k_{\mathrm{B}} T[c \ln c+(1-c) \ln (1-c)+\Omega c(1-c)],
\end{aligned}
$$

where $g_{\mathrm{Li}}$ and $g_{\mathrm{Si}}$ are the free energy densities of $\mathrm{Li}$ and $\mathrm{Si}$, respectively, $k_{\mathrm{B}}$ is the Boltzmann constant, $T$ is the absolute temperature and $\Omega$ is the dimensionless bond strength measured by the thermal energy $k_{\mathrm{B}} T$. To estimate the interface thickness, the derivative of the free energy with respect to $c$ is calculated as:

$\frac{\partial g}{\partial c}=-g_{\mathrm{Li}}+g_{\mathrm{Si}}+\Lambda k_{\mathrm{B}} T\left[\ln \frac{c}{1-c}+\Omega(1-2 c)\right]$.

Figure 3 shows the influence of bond strengths and the free energy of Si electrode on the free energy density and its derivative with respect to $c$. The changes in parameters $\Omega$ and $c$, show small effects on the SEI thickness. This indicates the changes will likely make no difference to the order of magnitude of the free energy density. When $\Omega>2, g$ is a double-well potential function, whereas there is a convex function for $\Omega<2$. Substitution of eq. (15) into eq. (12) yields

$\Lambda k_{\mathrm{B}} T \nabla^{2}\left(\ln \frac{c}{1-c}-2 \Omega c\right)=2 h_{0} \nabla^{4} c$,

which is the equilibrium equation for the concentration $c$. Based on eq. (16), the SEI thickness can be analyzed with the order-of-magnitude estimate.

A stable Li-ion concentration profile of the 1D binary system can be described $[34,43]$, using the Cahn-Hilliard phasefield model, as:

$\frac{c-c_{\mathrm{c}}}{\Delta c_{\mathrm{e}}}=\tanh \left(\frac{2 x}{l}+C\right)$,

where $c_{\mathrm{c}}=\left(c_{\mathrm{a}}+c_{\mathrm{b}}\right) / 2$ is the critical concentration, $\Delta c_{\mathrm{e}}=\left(c_{\mathrm{b}}-c_{\mathrm{a}}\right) / 2$ is the equilibrium concentration, $l$ is the thickness of the interface and $C$ is an integral constant. The distribution of the particle concentration in the 1D binary system exhibits a hyperbolic tangent model in the interface. Figure 4 shows a typical interfacial concentration profile.

A geometrical method is used to determine the SEI thickness with the concentration profile. Figure 4 plots a typical

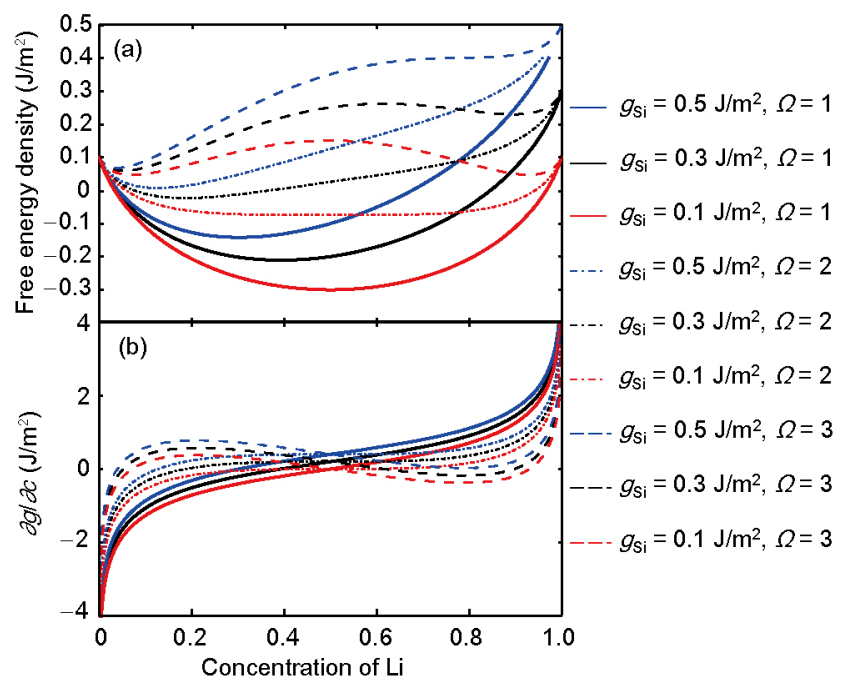

Figure 3 (Color online) (a) The curves of the free energy density $g$ with different bond strengths $\Omega$ and different Si electrodes $g_{\mathrm{Si}}$; (b) the curves of derivative of free energy density with respect to molar fraction with different bond strengths $\Omega$ and different Si electrodes $g_{\mathrm{Si}}$.

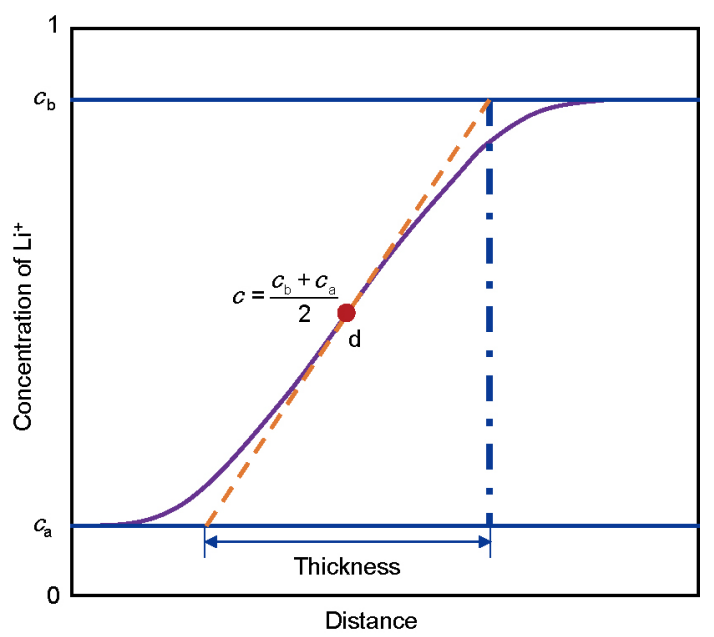

Figure 4 (Color online) Schematic of the Cahn-Hilliard finite thickness diffusion interface model. The orange dashed line represents the tangential of the curve.

interfacial concentration profile. The tangent of the curve is plotted at the d-point where the interface concentration is $c_{\mathrm{c}}$, and the interface thickness is calculated using the trigonometric tangent of the particle concentration distribution as shown in Figure 4. 


\subsection{Order-of-magnitude estimate}

The magnitude of the SEI thickness is estimated using eq. (16). The increments of both the concentration and the terms in the parentheses on the left-hand side of the equation are evaluated with the Taylor series expansions. The terms in the parentheses and the concentration of eq. (16) can be expanded at $c=1 / 2$ as:

$\left\{\begin{array}{l}\ln \frac{c}{1-c}-2 \Omega c=-\Omega+\left(\frac{1}{4}-\Omega\right) \Delta c+O\left[(\Delta c)^{2}\right], \\ c=\frac{1}{2}+\Delta c+O\left[(\Delta c)^{2}\right],\end{array}\right.$

where the big $O$ notation represents the limiting behavior of a function, and $f(x) \sim O(x)$ represents that $f(x)$ has the same growth rate as $x$. The constants $1 / 4,1 / 2$ and $\Omega$ are $O(1)$, so that $(1 / 4-\Omega) \Delta c$ in eq. (18) can be described as $O(\Delta c)$. Substituting all the above relations into eq. (18), one has

$\ln \frac{c}{1-c}-2 \Omega c \sim O(1)+O(\Delta c)$,

$c \sim O(1)+O(\Delta c)$

and taking eqs. (19) and (20) into eq. (16), the following approximate expressions on the left-hand and right-hand sides of eq. (16) are calculated, respectively. Noting that the derivative of a constant is zero, there are

$\Lambda k_{\mathrm{B}} T \nabla^{2}\left(\ln \frac{c}{1-c}-2 \Omega c\right) \sim \Lambda k_{\mathrm{B}} T \frac{\Delta c}{l^{2}}$,

$2 h_{0} \nabla^{4} c \sim h_{0} \frac{\Delta c}{l^{4}}$,

where

$\left\{\begin{array}{l}\Lambda k_{\mathrm{B}} T \nabla^{2}[O(1)+(\Delta c)] \sim \Lambda k_{\mathrm{B}} T \frac{\Delta c}{l^{2}}, \\ h_{0} \nabla^{4}[O(1)+O(\Delta c)] \sim h_{0} \frac{\Delta c}{l^{4}},\end{array}\right.$

and substituting eqs. (21) and (22) into eq. (16), one has

$\Lambda k_{\mathrm{B}} T \sim \frac{h_{0}}{l^{2}}$,

which indicates that the driving potential and the energy barrier are of the same order. The order-of-magnitude estimate of the SEI thickness is obtained straightforward from eq. (24) as:

$l \sim \sqrt{\frac{h_{0}}{\Lambda k_{\mathrm{B}} T}}$,

where the scaling law of the SEI thickness $l$ is derived. The scaling law $l \sim 1 / \sqrt{\Lambda}$ of the theoretical model is consistent with that of DA, meanwhile, eq. (25) gives an explicit relation between the thickness $l$ and the dimensionless number $h_{0} / k_{\mathrm{B}} T$ of the indefinite function $f$. Even though a rough order-of-magnitude estimate considers the leading terms only in the analysis, it may provide an important reference for the investigation of the SEI thickness.

Different anodes and electrolytes have influence on the initial SEI thickness via the energy barrier. The influence is depicted in Figure 5, which indicates that the SEI thickness increases with increasing energy barrier. As electrolytes and anodes change, the compositions of the SEI are different. For the same $\mathrm{Si}$ anode, the electrolyte with ethylene carbonate (EC) only will generate a SEI mainly composed by $\left(\mathrm{CH}_{2} \mathrm{OCOOLi}\right)_{2}$ whose energy barrier is low. The electrolyte composed by EC and diethyl carbonate (DEC) will produce a thicker SEI with a high energy barrier, whose main components are $\mathrm{C}_{2} \mathrm{H}_{5} \mathrm{COOLi}$ and $\mathrm{Li}_{2} \mathrm{CO}_{3}$.

The SEI thickness vs. temperature curves are plotted in Figure 6. It is crystal clear that the SEI thickness decreases monotonically with increasing temperature. As a result, high temperature will lead to a thin and unstable SEI. Thus, to form a dense and stable SEI, a low temperature is necessary. This result provides a reference for the working conditions of commercial Li-ion batteries.

\subsection{Estimation of the SEI thickness}

As the order-of-magnitude estimate has been done in the above section, a specific sample will be given to validate the estimation the SEI thickness. We assume that the electrolyte compositions have EC and DEC. To simplify the theoretical analysis, the following reaction on the surface of the electrode occurs

$\mathrm{EC}+\mathrm{e}^{-}+\mathrm{Li}^{+}=\mathrm{Li}_{2} \mathrm{CO}_{3}$,

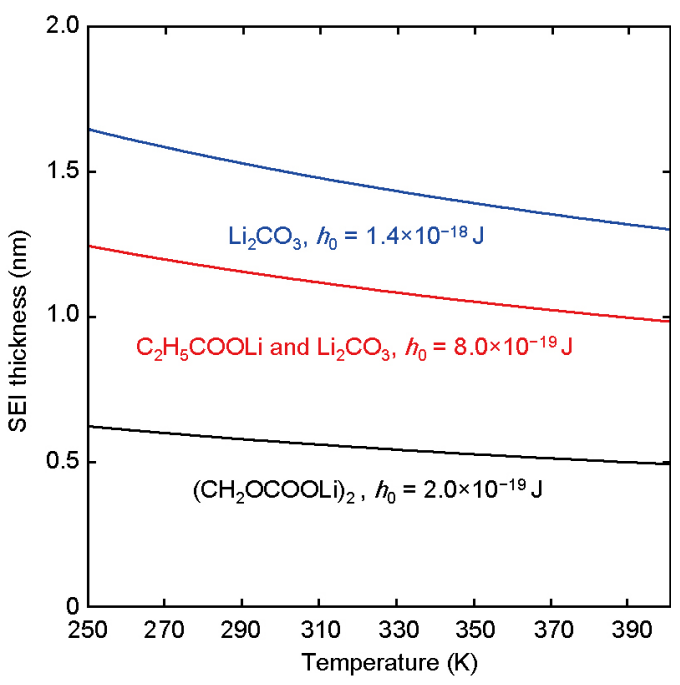

Figure 5 (Color online) The SEI thickness as a function of temperature with different energy barrier. The energy barrier changes with the different main compositions. 


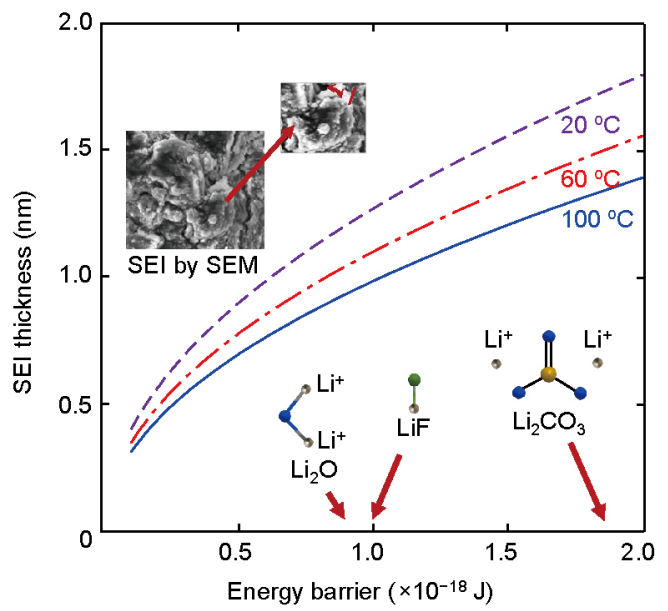

Figure 6 (Color online) The SEI thickness as a function of energy barrier with different temperature. The molecules are the main compositions of the SEI, which are produced by the reactions at the electrode-electrolyte interface. The SEI morphology by SEM is taken from ref. [30].

and using the electrochemical potential of the electrode reaction, the energy barrier $h_{0}($ at $300 \mathrm{~K}$ ) and the average number density $\Lambda$ are given as [44]:

$\left\{\begin{array}{l}h_{0}=\mu_{\text {anode }}-\mu_{\mathrm{Li}_{2} \mathrm{CO}_{3}}=1.88 \times 10^{-18} \mathrm{~J}, \\ \Lambda=1.569 \times 10^{20} \mathrm{~m}^{-2}-2.734 \times 10^{20} \mathrm{~m}^{-2},\end{array}\right.$

consequently, for simplicity and without loss of generality, the number density is assumed as $2.16 \times 10^{20} \mathrm{~m}^{-2}$. Substituting these quantities into eq. (25), one finds the estimation of the SEI thickness as:

$$
\begin{aligned}
& l \sim \sqrt{\frac{1.88 \times 10^{-18} \mathrm{~J}}{2.16 \times 10^{20} \mathrm{~m}^{-2} \times 4.14 \times 10^{-21} \mathrm{~J}}} \\
& \sim 1.45 \times 10^{-9} \mathrm{~m},
\end{aligned}
$$

by which the thickness SEI on the Si electrode surface is up to several nanometers. To the best of the authors' knowledge, there is no report about the SEI thickness on the Si electrode surface by direct observation. Thus, MD simulation will be used to verify the results of the order-of-magnitude estimate.

\subsection{The results of MD simulation}

To compare the results of magnitude estimation with the MD simulation, the numerical density distribution of Li-ion diffusion of the interface was simulated, as shown in Figure 7. Following the analysis of the thickness in Figure 4, the tangents of the midpoints $d_{1}$ and $d_{2}$ of Li-ion concentration profiles were given in the two interfaces. The two tangent lines (blue dashed lines in Figure 7) intersect with the concentration extension cords of $\mathrm{Si}$ and Li bulk phases (red dot-dashed lines in Figure 7), respectively. Using the trigonometric tangent function, the thicknesses of the two interfaces are 1.42 and $1.39 \mathrm{~nm}$, respectively.

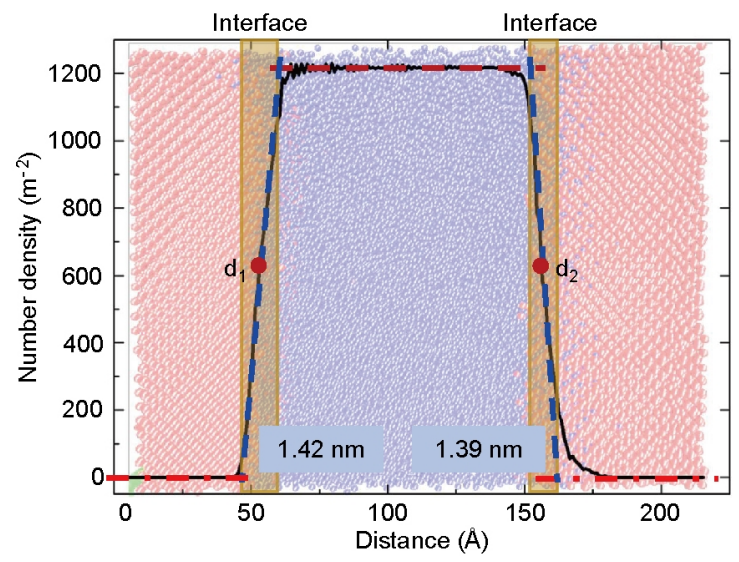

Figure 7 (Color online) The SEI thickness calculation by MD simulation. The black solid line is the curve of the numerical density distribution of Li-ion in the interface, the blue dashed lines are the tangent lines of the mid-points of the numerical density, and the red dot-dashed lines are the concentration extension cords. The yellow boxes are the interfacial zones.

The SEI thickness difference on the Si electrodes is explained as follows. The vacuum layers at the interface of the two Si electrodes emerge, using the periodic boundary condition. Then the vacuum layers interact with the cut-off radius of the 2NN MEAM potential function. The interaction causes the nonuniformity of the thickness of the two interfaces. The relative error only about $2 \%$ caused by the boundary asymmetry can be ignored. The nanoscale SEI films have been obtained using the MD simulation. Comparing with the order-of-magnitude estimate, we conclude that the SEI thickness of MD simulation is of the same order of that of the Cahn-Hilliard phase-field model.

\section{Conclusions}

In this paper, the order-of-magnitude of the SEI thickness on the Si electrode is estimated using both the Cahn-Hilliard phase-field model and MD simulation. The main conclusions are summarized as follows:

(1) a rough relation between the SEI thickness $l$, the number density $\Lambda$, the energy barrier $h_{0}$ and the temperature $T$ is derived using the DA, which gives the dimensionless thickness $l \sqrt{\Lambda}$ is a function $f$ of dimensionless numbers $h_{0} / k_{\mathrm{B}} T$, $c$ and $\Omega$;

(2) using the phase-field model for the further research, the initial SEI thickness analyzed and estimated in magnitude is about several nanometers. And the SEI thickness is in direct proportion to the square root of the energy barrier $h_{0}$ and in inverse proportion to the square root of the number density $\Lambda$ and the temperature $T$.

(3) the distribution curve of Li-ion concentration is obtained by MD simulation. The SEI thickness also in nanoscale is derived using the simulation. The SEI thickness based on MD simulation is in good agreement with that of 
the theoretical model.

The established model is proved quite useful and effective to estimate the SEI thickness. This study will help in better understanding the formation mechanism of the SEI and the influence of relevant physical quantities on the SEI thickness.

This work was supported by the National Natural Science Foundation of China (Grant Nos. 11372313, U1562105, and 11611130019), the Chinese Academy of Sciences (CAS) through CAS Interdisciplinary Innovation Team Project, the CAS Key Research Program of Frontier Sciences (Grant No. QYZDJ-SSW-JSC019), and the CAS Strategic Priority Research Program (Grant No. XDB22040401).

1 Y. Zhao, J. Feng, X. Liu, F. Wang, L. Wang, C. Shi, L. Huang, X. Feng, X. Chen, L. Xu, M. Yan, Q. Zhang, X. Bai, H. Wu, and L. Mai, Nat. Commun. 5, 4565 (2014).

2 F. Q. Yang, Sci. China-Phys. Mech. Astron. 59, 114611 (2016)

3 J. S. Kim, and Y. T. Park, J. Power Sources 91, 172 (2000).

4 B. Peng, Y. L. Xu, K. Liu, X. Q. Wang, X. H. Shi, and F. M. Mulder, Sci. China-Phys. Mech. Astron. 60, 064611 (2017).

5 P. Verma, P. Maire, and P. Novák, Electrochim. Acta 55, 6332 (2010).

6 E. Peled, and D. Golodnitsky, SEI on Lithium, Graphite, Disordered Carbons and Tin-Based Alloys (Imperial College Press, London, 2004), pp. 1-59.

7 D. Aurbach, and A. Zaban, J. Electroanal. Chem. 348, 155 (1993).

8 L. Fransson, T. Eriksson, K. Edström, T. Gustafsson, and J. O. Thomas, J. Power Sources 101, 1 (2001).

9 I. Epelboin, M. Froment, M. Garreau, J. Thevenin, and D. Warin, J. Electrochem. Soc. 127, 2100 (1980).

10 D. Aurbach, and Y. Eineli, J. Electrochem. Soc. 142, 1746 (1995).

11 S. K. Jeong, M. Inaba, R. Mogi, Y. Iriyama, T. Abe, and Z. Ogumi, Langmuir 17, 8281 (2001).

12 Y. Domi, T. Doi, H. Nakagawa, T. Yamanaka, T. Abe, and Z. Ogumi, J. Electrochem. Soc. 163, A2435 (2016).

13 M. Winter, and P. Novák, J. Electrochem. Soc. 145, L27 (1998).

14 Z. Ogumi, A. Sano, M. Inaba, and T. Abe, J. Power Sources 97, 156 (2001).

15 P. Jara-Ulloa, P. Salgado-Figueroa, R. Moscoso, and J. Arturo Squella, J. Electrochemical Soc. 160, H243 (2013).

16 D. Aurbach, B. Markovsky, I. Weissman, E. Levi, and Y. Ein-Eli, Electrochim. Acta 45, 67 (1999).

17 D. Aurbach, M. D. Levi, E. Levi, H. Teller, B. Markovsky, G. Salitra, U. Heider, and L. Heider, J. Electrochem. Soc. 145, 3024 (1998).

18 D. Aurbach, Y. Ein-Eli, O. Chusid, Y. Carmeli, M. Babai, and H. Yamin, J. Electrochem. Soc. 141, 603 (1994).

19 D. Aurbach, J. Power Sources 89, 206 (2000).

20 D. Aurbach, M. L. Daroux, P. W. Faguy, and E. Yeager, J. Electrochem. Soc. 134, 1611 (1987).

21 I. Warshawsky, J. Electrochem. Soc. 127, 1324 (1980).

22 S. P. Kim, A. C. T. Duin, and V. B. Shenoy, J. Power Sources 196, 8590 (2011).

23 K. Xu, Energies 3, 135 (2010).

24 C. K. Chan, R. Ruffo, S. S. Hong, and Y. Cui, J. Power Sources 189, 1132 (2009).

25 P. Zuo, and Y. P. Zhao, Extreme Mech. Lett. 9, 467 (2016).

26 H. Ji, J. W. Kim, Y. E. Sung, S. M. Oh, Electrochem. Solid-State Lett. 7, A306-A309 (2004)

27 P. Zuo, and Y. P. Zhao, Phys. Chem. Chem. Phys. 17, 287 (2015).

28 K. W. Schroder, A. G. Dylla, S. J. Harris, L. J. Webb, and K. J. Stevenson, ACS Appl. Mater. Interfaces 6, 21510 (2014).

29 J. L. Zang, and Y. P. Zhao, Int. J. Eng. Sci. 61, 156 (2012).

30 J. L. Zang, Electrode Surface Diffusion Induced Stress Evolution and
Anti-Pulverization in Nanostructured Si-Based Anode for Lithium Ion Batteries, Dissertation for the Doctoral Degree (University of Chinese Academy of Sciences, Beijing, 2013).

31 C. Pereira-Nabais, J. Światowska, A. Chagnes, F. Ozanam, A. Gohier, P. Tran-Van, C. S. Cojocaru, M. Cassir, and P. Marcus, Appl. Surface Sci. 266, 5 (2013).

32 J. W. Cahn, J. Chem. Phys. 42, 93 (1965).

33 Y. P. Zhao, Physical Mechanics of Surfaces and Interfaces (Science Press, Beijing, 2012).

34 W. Lu, and Z. Suo, J. Mech. Phys. Solids 49, 1937 (2001).

35 Z. Suo, and W. Lu, J. Nanoparticle Res. 2, 333 (2000).

36 J. W. Cahn, Acta Metall. 9, 795 (1961).

37 Y. P. Zhao, Nano and Mesoscopic Mechanics (Science Press, Beijing, 2014).

38 S. Nosé, J. Chem. Phys. 81, 511 (1984).

39 B. J. Lee, and M. I. Baskes, Phys. Rev. B 62, 8564 (2000).

40 B. J. Lee, Calphad 31, 95 (2007).

41 Z. Cui, F. Gao, Z. Cui, and J. Qu, J. Power Sources 207, 150 (2012).

42 Z. Cui, F. Gao, Z. Cui, and J. Qu, Modelling Simul. Mater. Sci. Eng. 20, 015014 (2012).

43 J. W. Cahn, and J. E. Hilliard, J. Chem. Phys. 28, 258 (1958).

44 H. J. Ploehn, P. Ramadass, and R. E. White, J. Electrochem. Soc. 151, A456 (2004).

\section{Appendix Dimensional analysis of the SEI thickness}

Considering the diffusion model of a binary system, the SEI forms when the balance of driving energy and energy barrier is reached. The physical quantities to work with this equilibrium process are the SEI thickness $l$, the energy barrier $h_{0}$, the number density $\Lambda$, the thermal energy $k_{\mathrm{B}} T$, the concentration $c$ (dimensionless) and the dimensionless bond strength $\Omega$. Dimension is used to express the essential nature of a quantity. The dimensions of these physical quantities are

$\left\{\begin{array}{l}{[l]=L,} \\ {[\Lambda]=L^{-2},} \\ {\left[k_{\mathrm{B}} T\right]=E,} \\ {\left[h_{0}\right]=E,}\end{array}\right.$

where $L$ is the dimension of length, $E$ is the dimension of energy and $[*]$ represents the dimension of a physical quantity. There are six physical quantities depending on two independent dimensions. We can get the following function by Buckingham theorem as:

$l=\frac{1}{\sqrt{\Lambda}} f\left(\frac{h_{0}}{k_{\mathrm{B}} T}, c, \Omega\right)$,

where the SEI thickness correlates with a dimensionless function $f$ of the dimensionless numbers $h_{0} / k_{\mathrm{B}} T, c$ and $\Omega$. There is an inverse correlation between the SEI thickness and the square root of number density $\Lambda$. However, the function $f$ has no concrete form and there are implicit relations of $h_{0} / k_{\mathrm{B}} T$, $c, \Omega$ and $l \sqrt{\Lambda}$. Thus, we need to do a further analysis with the theoretical model of the SEI thickness. 